Erschienen in: IS\&T International Symposium on Electronic Imaging 2019 : Stereoscopic Displays and

Applications XX. - Springfield, VA : Society for Imaging Science and Technology, 2019. - S. 641-1 - 641-15

https://doi.org/10.2352/ISSN.2470-1 173.2019.3.SDA-641

This work is licensed under the Creative Commons Attribution 4.0 International License.

To view a copy of this license, visit http://creativecommons.org/licenses/by/4.0/.

\title{
Tiled Stereoscopic 3D Display Wall - Concept, Applications and Evaluation
}

\author{
Björn Sommer 1,2,\$, Alexandra Diehl' ${ }^{1}$,Michael Aichem ${ }^{1}$, Philipp Meschenmoser ${ }^{1}$, Kim Rehberg$^{1}$, David Weber ${ }^{1}$, Ying Zhang $^{1}$, \\ Karsten Klein ${ }^{1,3}$, Daniel Keim ${ }^{1}$, Falk Schreiber ${ }^{1,3}$ \\ 1 University of Konstanz, Konstanz, Germany \\ ${ }^{2}$ Royal College of Art, London, UK \\ ${ }^{3}$ Monash University, Melbourne, Australia \\ \$bjoern@CELLmicrocosmos.org
}

\begin{abstract}
The Tiled Stereoscopic 3D Display Wall (TS3DW) is a monitor system consisting of six consumer $3 D$ TVs. Two monitors reside on a mobile display mount. One standard configuration is to use them in a 135-degree angle to each other, having one mobile mount in the center, and one at each side. In this way, the system can be transported to multiple locations across a campus as well as used in different application scenarios. This system was already used for a number of research projects and presentations.

In this work, we present the concept, applications and evaluation of the implemented system. First, we will discuss the hardware setup, the passive circular polarization technology provided by the LG $3 D T V$ s and its limitations. Then, two application cases making use of Stereoscopic $3 D$ visualization will be discussed and compared to previous work:

- Visualization and Analysis of Bird Trajectories,

- Visualization and Analysis of Meteorite Data.
\end{abstract}

Finally, we discuss a comprehensive evaluation of the system and its stereoscopic capabilities featuring 16 participants with different body heights. Three major questions were evaluated:

- Is TS3DW an appropriate environment for group presentations?

- If so, which aspects have to be taken into account during its configuration?

- Does TS3DW show potential to be used in the context of static and/or dynamic bird visualization?

\section{Introduction}

Display walls, consisting of a number of monitors or TVs, are often used in companies or universities for multiple purposes: high resolution displays, group presentations, large-scale touch interaction, collaborative data analysis etc. As long as only 2D projections are used, the configuration of these display environments is relatively easy - from a hardware as well as software perspective. But in case Stereoscopic 3D (S3D) visualization should be used, things are getting complicated, as different screens have to be combined, synchronized, and the maintenance of an optimal stereoscopic vision has to be provided to the viewers.

S3D display devices are being developed since many decades. One of the milestones of developing S3D display environments is the CAVE (CAVE Automatic Virtual Environment) introduced in the 1990s [1]. Usually - especially in the past - a number of back projection systems are used to create a stereoscopic display environment using different configurations of projection screens - e. g. front, left and right side, floor and/or ceiling. Each of these projection screens is normally associated with two projectors for the left and right perspective. Since the 1990s, many universities and companies adapted this display setup. Just a few years ago the next iteration, CAVE2 $®$ was introduced which was creating a nearly $360^{\circ}$ experience by combining arrays of stereoscopic monitors to a high-resolution stereoscopic display [2].

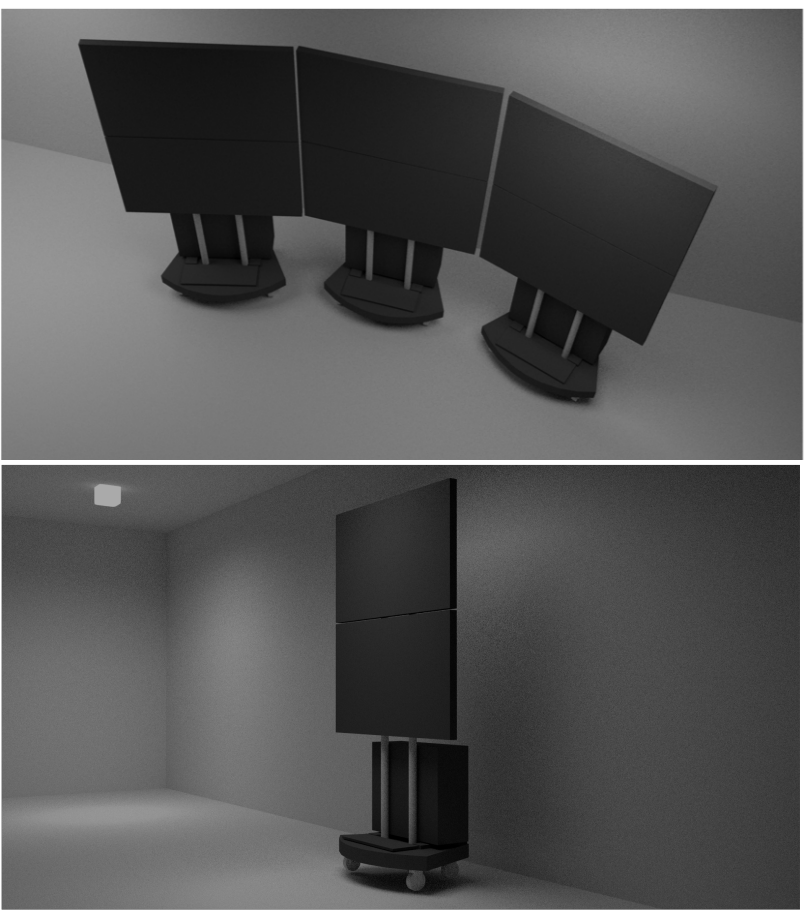

Figure 1. Initial sketches of the Tiled S3D Display Wall modelled with Blender [3]. The mobile stands are already visible combined with two 55" displays per row placed on top of each other. Top: three two-monitor systems side-by-side, Bottom: one two-monitor system including the computer placed on top of the mobile platform.

The main problem of these configurations is the fact that they are quite demanding in terms of financial and human resources investment. In addition, the advent of affordable Head- 
Mounted Display (HMD) devices like the Oculus Rift $\AA$ or HTC

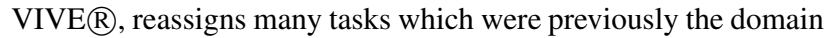
of CAVEs to HMDs [4]. However, group presentations are still an important domain of CAVEs [5].

Another CAVE-inspired installation is the StarCAVE of the University of California in San Diego - which is still based on a projector-based back projection system - and its successor, the SunCAVE, which is based on a number of consumer-based S3D LCD TVs [6]. The visitor is entering a display setup which is surrounding him by $360^{\circ}$-similar construction.

Starting from a pentagon-shaped floor layout, five wall segments are divided into three articulated display surfaces. This construction results in fifteen wall projection surfaces plus a floor surface. Each of them is driven by stereoscopic back projection systems. The displays are placed on top as well as on the bottom in an optimized angle, approximating an equidistant viewer-toscreen configuration.

A quite different approach - also from the University of California in San Diego - is the WAVE Display, created by Greg Dawe, Jürgen Schulze, et al. in 2013. A wave-like configuration of different S3D TVs should provide an optimal viewerdisplay alignment consisting of five columns and seven rows of 3D TVs. The construction of WAVE was supported by a multimillion grant. Please consult the following paper for additional potential CAVE-like configurations [7].

In this work we explore a more affordable approach for group presentation: a Tiled Stereoscopic 3D Display Wall $(T S 3 D W)$. By using consumer grade S3D displays, the overall costs of the system can be lowered to not more than 25,000 EUR. In comparison to approx. 2 million EUR required for a CAVE2 it is an interesting alternative for small groups.

Here, we are exploring the applicability of this display device to group presentations. First, we are introducing two application cases and discuss the usage of a stereoscopic display setting in their context.

- Bird visualization: analysis of bird movement over long distances

- Meteor visualization: fireball and meteorite analysis using globe-based visualization

\section{Bird Tracking and Ornithology}

Due to recent advances in GPS sensor technology, a large amount of animal movement data is currently collected for use in collective behavior research [8]. Analysis of such data can help in a range of areas, such as better understanding of the mechanisms behind animal decision making, investigating the effects of largescale events, such as natural disasters, and better explaining the factors behind disease dynamics like bird flu. However, the analysis is challenging, as the interpretation of movement needs to be put in the context of the environment in which it happened, among others the geography, season, weather, further environmental factors like vegetation, and individual characteristics of the animals and perhaps the composition and behavior of the group it belongs to. In addition, results from analysis methods that can help elucidate roles and behavior in a group need to be integrated in the analysis workflow. S3D display walls provide the screen estate and the resolution to support the visual analysis of such a combination of abstract and spatial data, and allow to compare the behavior of different individuals or groups, or to show different aspects and perspectives at the same time. In particular, watching animals move in $3 \mathrm{D}$ improves the assessment of spatial relationships that are important for the analysis [9, 10, 11]. Such a visual analysis greatly helps to gain insight into the data, to check its consistency, and it supports the investigation of the environmental influence factors underlying animal decision making.

The data under investigation is usually collected from GPS sensors with which the animals are tagged, and which can differ in the measured data, its temporal resolution and precision, and a minimal set of measured data consists of series of positions (longitude, latitude and possibly altitude for bird movements) together with a time stamp for each position. The data used in our study was obtained from the Movebank data repository, a large database of animal movement data sets [12].

Alternative immersive approaches to visualize bird-related data are discussed in [13].

\section{Meteors and Astrostatistics}

The use of S3D display walls opens new opportunities for the evaluation of analytical 3D visualization tools for spatial structures. The use of S3D technology in the context of meteor visualization could be particularly useful for Astrostatistics researchers, who need tools that can assist them to solve problems and answer questions related to trends detection in time and space, hotspots detection, hidden correlations, anomaly detection, as well as other spatial statistical features.

Here, we present two types of meteor visualizations; fireballs and meteorites. A fireball is a meteor that appears at least as bright as the planet Venus in the night sky [14]. A meteorite is a fragment of a meteoroid or an asteroid that survives, passes the atmosphere and hits the ground [15].

Previous work focused on the use of 3D views and stereoscopic displays to recreate the space, the solar system, and the Milky Way. The work of Sales Dias et al. [16] presented Galactica, a digital planetarium that proposes the use of stereoscopy to depict photorealistic scenes in a high-resolution multi-display projection system. The World Wide Telescope (WWT) presented for the first time by Alexander Szalay and Jim Gray in 2001 [17], visualizes astronomical, Earth and planetary data. It allows the user to create and perform educational tours through the solar system and imagery of the Universe. Stellarium is an open-source astronomy software developed by Chéreau et al. [18] in 2001 that visualizes the sky and its celestial objects from different coordinates and panoramas. It is possible to inspect the sky from the Earth or from other celestial objects. They also provide stereoscopic functionality that could be used for similar purposes like our applications. We decided to use our own implementation to have a controlled setup and evaluation environment to compare different application scenarios. The main difference between the aforementioned tools and our work is that the former have a focus on the realistic representation of spatial objects with the aim of reproducing immersive and virtual reality experiences, while our work focuses on the abstract representation of spatial objects to perform data analysis.

More aligned to our work are several well-known 2D and 3D non-immersive visualization tools that present and visualize quantitative features of the spatial objects. Asterank [19] is a scientific and economic database of asteroids, enabling the user to visualize 
the solar system and over 600,000 asteroids. It provides a 3D visualization of information about the asteroids, such as mass, composition, and a value estimation from different sources. Stuff in Space [20] depicts an impressive and simple way of visualizing all objects put into space by humans orbiting the Earth, classified as: satellite, rocket body, and debris. It also provides different ways of visual interaction, such as picking a point in the globe, highlighting a specific orbit, filtering objects, and zooming in and out. Meteor Showers [21] visualizes the Solar system and the meteor showers hitting the Earth. It provides filters and additional information that the users can query about specific meteor showers' orbits. BOLIDES [22] is an interactive timeline visualization that depicts the history of meteorites observed when falling and hitting the ground. The data is collected from the Meteoritical Bulletin Database containing information about the name of the meteorite (identification), location, type, and mass. The main difference between those previous approaches and ours is that we make use of immersive technologies and 3D stereoscopic displays to enhance the analytical tasks.

\section{From Concept to Implementation}

First, the concept and hardware setup of the Tiled S3D Display Wall is introduced, and then, based on this construction, two software application based on the previously discussed domains are introduced.

\section{Tiled Stereoscopic 3D Display Wall}

The initial concept of the Tiled Stereoscopic 3D Display Wall $(T S 3 D W)$ is shown in Fig. 1 From the beginning, it was an important aspect of our system that it can be used for $2 \mathrm{D}$ and $3 \mathrm{D}$ presentations as well. Therefore, articulated display surfaces configuration like the one of the aforementioned Wave or the SunCAVE were not an option.

Three display columns with two displays had to be created. The columns had to be mobile to transport them inside the university building and to change the positioning of the columns in relation to each other, e.g. for changing the angle between two columns. For this purpose, lockable castor wheels were installed. Each monitor column can be balanced towards the ground by using height-adjustable screws. Moreover, the height of every monitor can be altered along its column. In this way, monitors could also be moved vertically apart from each other.

The six 3D TVs - see Fig.2- are LG 55EF9509 55" displays (display height: $70.1 \mathrm{~cm}$ ). The 3D TVs are using OLED technology providing perfect black and very thin bevels. These monitors provide $4 \mathrm{~K}$ resolution in $2 \mathrm{D}$ mode, supporting vertical Full HD resolution (3840X1080) for each eye using the S3D mode. The stereoscopy effect is based on circular polarization. For this purpose, the user has to wear passive circularly polarized glasses delivered with the monitors. Glasses from many passive 3D cinema screen setups are often also be compatible. The use of passive glasses is more comfortable than the use of active glasses. However, the technology also comes with drawbacks. And these drawbacks will be evaluated in this work.

The LG 3D TVs were merchandised in Europe as "Cinema 3D" line. More precisely, the technology used by LG is called FPR (Film-type patterned retarder). This technology shows images intended for the left and right eye through different patterns in a circular polarizer. The direction of polarization (left-handed vs. right-handed) is altering line-based: "An FPR 3D display has even and odd rows polarized clockwise and counterclockwise, respectively" [23].

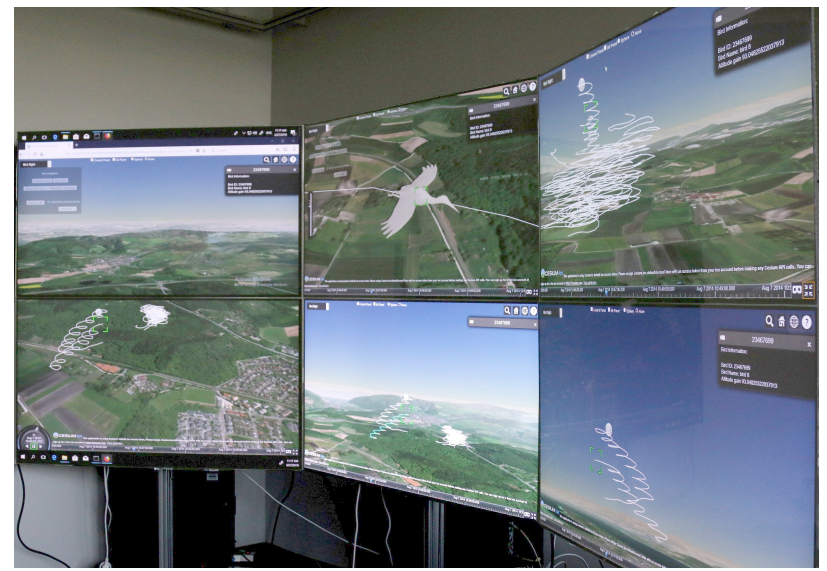

Figure 2. Monitor setup with different perspective views on the same bird flock data set.

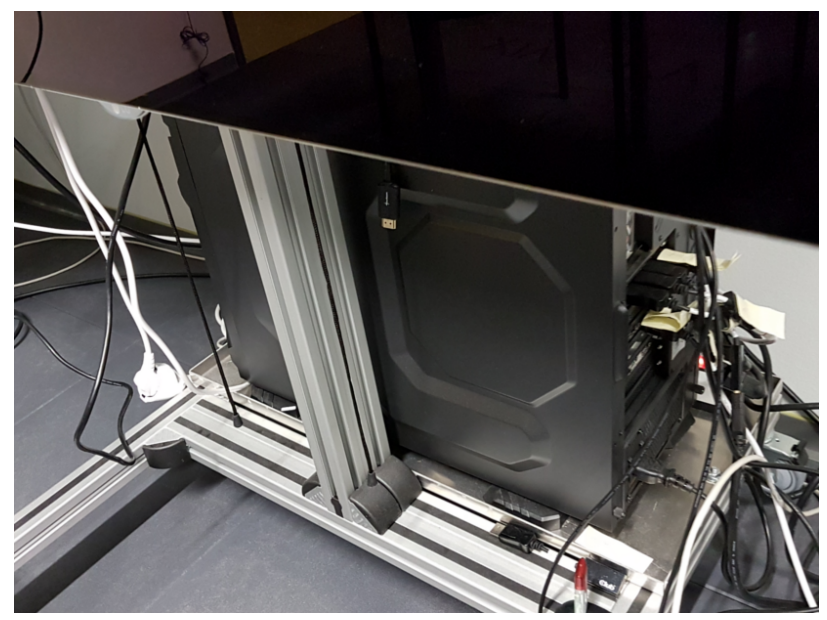

Figure 3. The computer connected to the TS3DW.

This is the reason why the vertical resolution of the $4 \mathrm{~K}$ display is bisected. In addition, the 3D effect is designed by LG to be optimal for a user sitting directly in front of the screen. Whereas the sitting position is relatively tolerant in horizontal direction, the vertical direction is very limited. If the user sitting in front of the screen rises and changes the position by, e.g., $50 \mathrm{~cm}$, the stereo effect is inverted as the angle of the eyes towards the monitor changes. Therefore, the 3D TV software as well as many 3D software players for PCs provide an option to invert the stereo effect.

We will show here that, although the $3 \mathrm{D}$ effect is inverted when drastically changing the vertical viewing position, there are strategies to combine different monitors and use them in groups consisting of upright standing people featuring heterogeneous body heights.

The Tiled 3D Display Wall is connected to a single computer based on an ASUS Z170 Pro Gaming mainboard with an Intel i7-6700 CPU and 128 GB Ram. The PC is equipped with 
two NVIDIA Quadro M4000 cards, as can be seen in Fig. 3. The graphics can be used in SLI mode. SLI mode was not used nor required for the evaluation done here as all TVs have been used as a separate Windows 10 desktop screen. Another big advantage of using all six monitors with a separate perspective is the fact that the S3D effect cannot be disturbed by the bevels of the monitors. This might be the case if all monitors are used as on big screen, showing a single perspective and the visualized 3D object lies between the projection plane and the near plane (for more details, please consult [11]).

\section{Bird Visualization with Cesium}

Our bird movement visualization is based on Cesium [24], a webbased open source framework for geospatial 3D map and globe visualizations. The basic framework Cesium is available from https://cesiumjs.org For this work, we were using Cesium 1.52. Our software can be run on a single machine or be hosted on a server and accessed by multiple client machines. Multiple visualizations are possible, and each visualization is rendered in a browser window.

As the original Cesium S3D implementation was developed towards mobile HMD usage, we had to modify Cesium to provide S3D functionalities compatible to large display walls. Especially the eye distance had to be changed. To overcome problems like crosstalk/ghosting [25] the user can change the distances between the stereo perspectives by using the GUI. Moreover, in stereo mode, the complete GUI is hidden to prevent disturbance of the S3D vision.

Another important aspect of the Tiled S3D Display Wall is that there is only a limited number of sweet spots, i. e., the position from which the stereoscopic vision is functional for each of the six screens. Similar approaches like the StarCAVE coped with this problem in a similar way [6]. In this case, the sweet spot is in the center in front of the screens.

\section{Meteor Visualization with Cesium}

The meteor visualization is also based on the Cesium Framework. Contrary to the bird movement visualization it makes use of only one perspective and therefore only needs a single display screen.

We selected two different data sources to visualize celestial objects with a focus on objects that enter the atmosphere and potentially hit the Earth: fireballs and meteorites. For the meteorite landings we used data presented by NASA and collected by the Meteorological Society. The meteorite landings contain valuable information about the material from which they are made that could help to identify where they come from. It also contains information about the mass in grams, the year of discovery, and whether it was found or observed.

For the fireballs we used data from user-observed events collected by the American Meteor Society (AMS) [15]. The data from the AMS consists of reports that members of the association make voluntarily. This kind of reports constitute a great opportunity to understand where people report the majority of the fireballs and how this correlates to other demographic factors. We visualized the meteorites as dots, encoding the mass using the area of the dots, and the color to identify its material class. For the fireballs, we visualized fireballs' trajectories and also the potential place of impact in case they (would) have reached the Earth.

We used a non-realistic visual encoding to avoid distractions

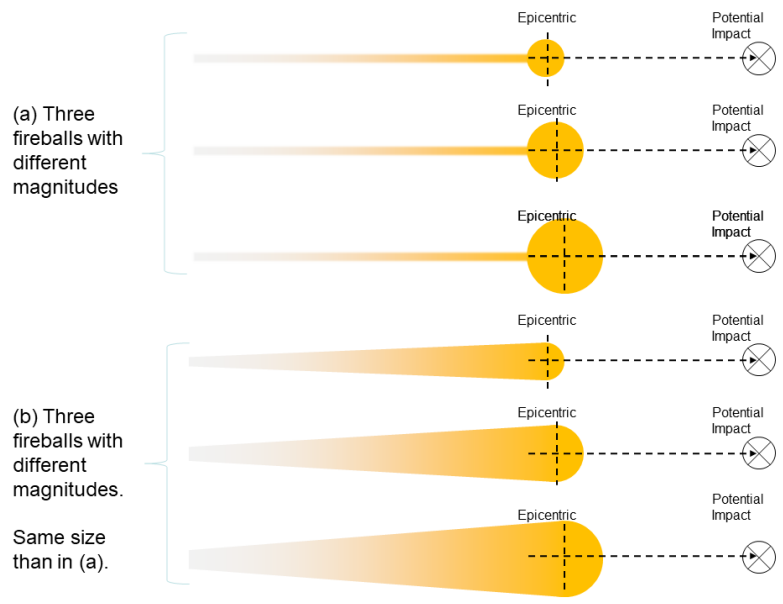

Figure 4. As part of our design process for the visualization of fireballs, we evaluated simplified designs that yet show all the information required for our analysis: stellar magnitude mapped as size, observed duration in time as a cylindrical line and extended trajectory as a dashed line connected to the potential place of impact, depicted as a crossed-dot on the ground, see also Figs. 105 and 17

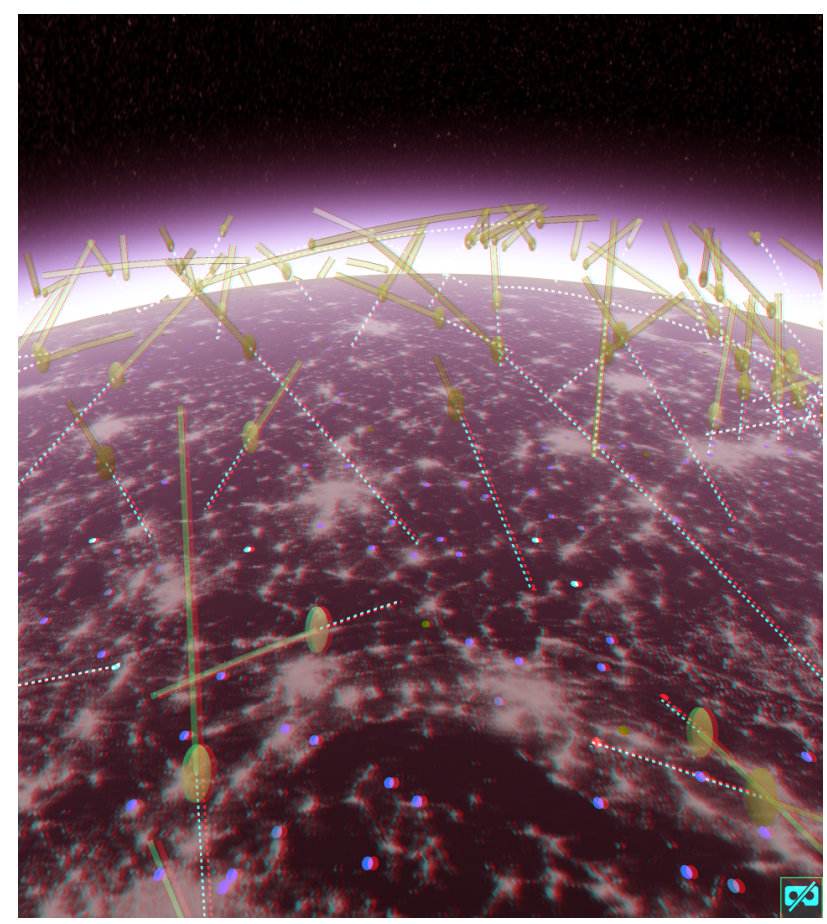

Figure 5. Close view of fireballs entering into the Atmosphere and meteors on the ground. The white dots on the ground represent potential fireball potential impacts. Meteorites are depicted as equally-sized dot with color encoding their composition as follows: Chondrite - blue, (Achondrite-cyan, Stony Iron (Pallasite and Mesosiderite) - green, Iron - magenta, unknown meteorite types - grey. Please use S3D red/cyan anaglyph glasses to see the stereo effect. 
on the analysis and mapped the Earth into a gray scale texture to avoid interference with the actual displayed data. We used the visual variables, size, and opacity to represent some of the most important meteor properties. The fireball was designed as a glyph that shows its duration in time as a trajectory, and extends the non-observed trajectory and potential place of impact as a dashedline trajectory, as shown in Fig. 4 Color was used to encode the average stellar magnitude of fireballs, a circular shape to show the start and end of fireball and a dashed line to depict its extended potential trajectory and place of impact. The meteorites where visualized as circular surfaces of fixed size on the ground. We use color coding to show the meteorite composition. The color scale for fireballs and meteorites are complementary in the color wheel to make a clear distinction between the two type of meteors.

Our Cesium web application can be found in:"'https://meteorite.dbvis.de/" - Proto3D

\section{Application Cases}

In the previous paragraphs we discussed how Cesium is applied to bird and meteor visualization. Here we discuss now how S3D visualization in particular is used to perform analytical tasks by introducing concrete scenarios.

\section{Bird S3D Visualization}

Large screens allow for either one large viewing area covering a huge landscape and allow putting the birds in a larger context, or can provide several views (in several browser tabs) in parallel on different screens, see Fig. 6. Here we use a multi-screen and multi-perspective arrangement with different view- and timepoints that can be customized depending on the user's preferences. An important aspect for use of S3D visualizations, in particular in a collaborative analysis, is the optimization of the sweet spot, such that all participants can have a similar viewing experience.

Here, we analyze a dataset of storks traveling close to the Lake Constance in August 2014. During their flight, they are taking advantage of thermals to gain altitude [26]. The corresponding data set can be found in the Movebank [27]. A typical scenario is shown in Fig. 7. Different views onto the data set in 3D mode. The white lines show the trajectory data recorded by the GPS senders. The lines are not smooth to enable the researchers to see the concrete GPS-recorded coordinates. Here, three exemplary multi-perspective scenarios are discussed, showing the potential of multi-monitor setups:

- Fig.7 The first scenario shows a single individual from six different perspectives: top, bottom, three side views from different angles, as well as one overview, showing the neighbourhood of the stork.

- Fig. 8 The second scenario shows three birds. For each bird, two perspectives are shown: the top perspective on the bottom screen, and the follower perspective on the top screen.

- Fig. 9. The third scenario observes five separate birds from back perspective. In the bottom center, the overview is showing all birds at once.

\section{Meteor S3D Visualization}

We used TS3DW to analyze several meteor characteristics, such as type, composition, mass, emitted energy, their trajectory and, in case of the fireballs, the angle of entrance to the Earth. TS3DW allows the analyst to observe meteors from different perspectives, assess their qualitative density, their trajectory, the angle of entrance to the atmosphere, and their trajectory travelling the Earth. To identify the angle of entrance of a meteor travelling the Earth could help to determine whether the meteor will explode high in the atmosphere due to friction or hit the Earth. The 3D geospatial views using latitude, longitude, and altitude can help to determine from where fireballs approach the Earth, for example, whether it is heading the Earth orbits, or whether it comes from behind the Earth [14]. Fig. 10 shows fireballs entering the atmosphere with different angles and at different geographic coordinates. On the ground, the potential impact of those fireballs is depicted as white circular surfaces. Also, meteorites can be seen in Fig. 5 and in Fig. 17 with a higher level of detail. Color is used to identify the meteorite types as follows:

- Chondrite - blue,

- Achondrite - cyan,

- Stony Iron (Pallasite and Mesosiderite) - green,

- Iron - magenta, and

- unknown meteorite types - green.

Figs. 10 and 17

For this application, we focus on the use of simple visual encoding of the spatial and temporal characteristics of meteors by using color coding, shape, and visualization of fireball trajectories and potential impact. Other interesting applications are the study of the composition of meteorites, their time frequency, and spatial distribution in order to determine the grade of hazardousness, as well as their economic value. It would be interesting to evaluate and measure how well analysts can identify hot spots of fireballs from several perspectives using color and density-based opacity. In future work, we plan to add more filters to query other attributes, such as composition, brightness, and duration.

\section{Evaluation}

By using the bird visualization, we evaluated the TS3DW setup. During our evaluation, three basic questions were addressed:

- Is TS3DW an appropriate environment for group presentations?

- If so, which aspects have to be taken into account during its configuration?

- Does TS3DW show potential to be used in the context of static and/or dynamic bird visualization?

Although we see value in using the system as a single user, we see much potential in the use of HMDs in the single-user domain. Therefore, from a system like TS3DW, we expect that it can be used for group presentations.

For the purpose of evaluating the usability in groups, the number of optimal viewing positions (sweet spots) had to be determined. For this purpose, the area in front of the TS3DW was subdivided into 18 different sections, as can be seen in Fig. 11 and Fig. 12 Each one of the squares is $0.5 \times 0.5 \mathrm{~m}^{2}$ in size. Each square was numbered from I. to XVIII. 


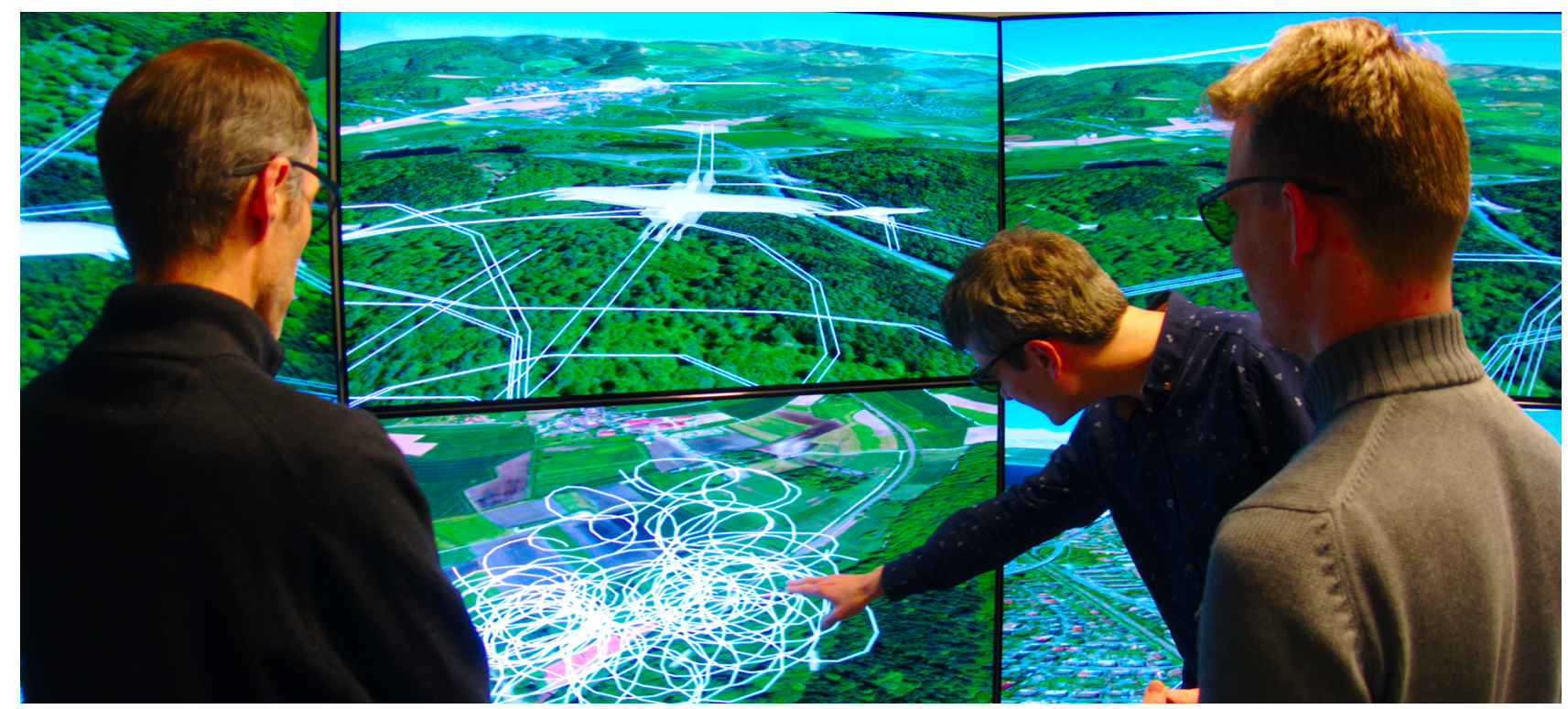

Figure 6. The monitor wall setup facilitates face-to-face collaborative analysis. The users are using 3D glasses compatible to the Tiled S3D Display Wall.

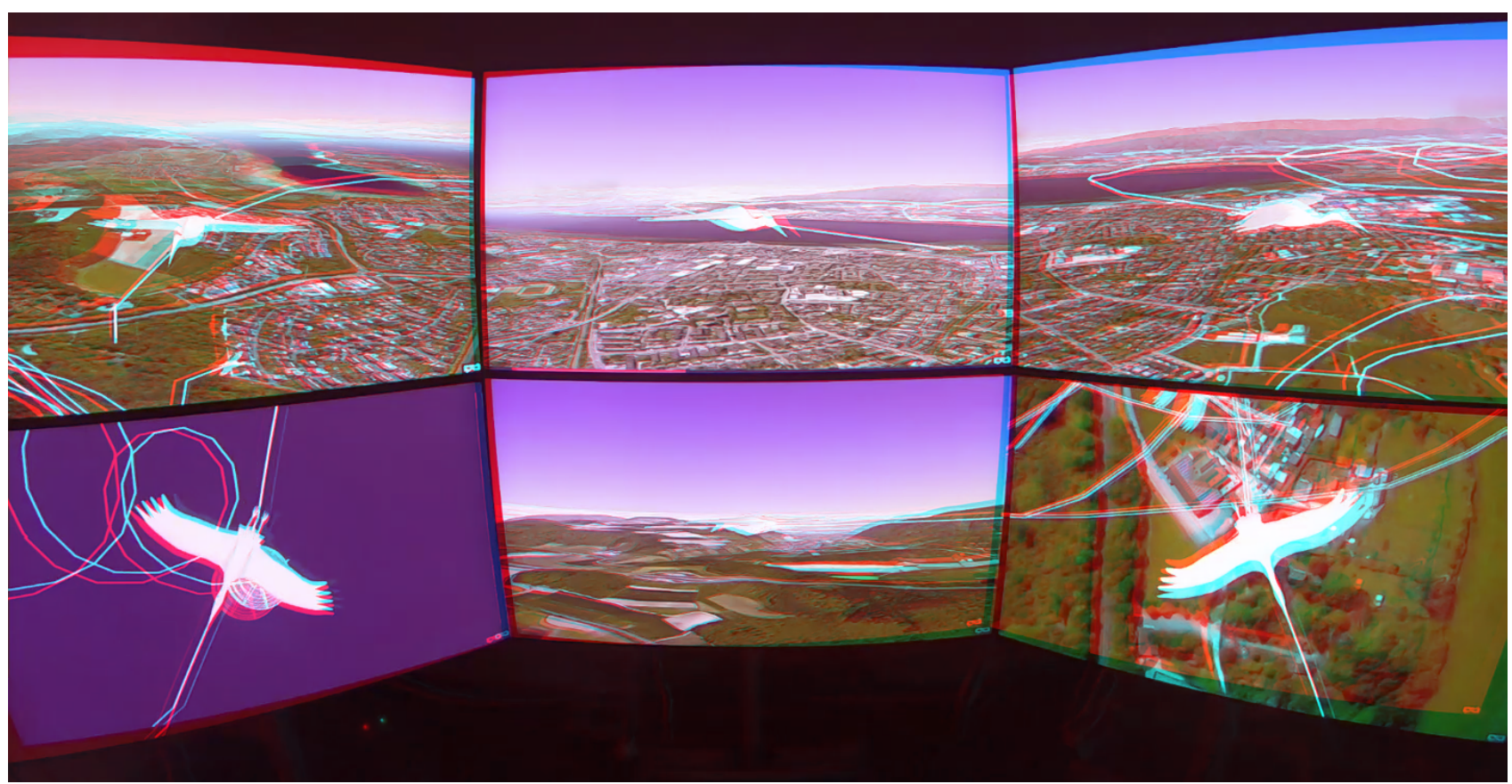

Figure 7. Bird Visualization - Scenario 1 Bird: $A$ single stork is shown from different perspectives: from left top to bottom right: $-45^{\circ}$ front, front, $+45^{\circ}$, bottom, back, top. All monitors are used here in 3D mode. The white lines show here the trajectory data recorded by the GPS senders. The lines are not smooth to enable the researchers to see the concrete GPS-recorded coordinates. Please use S3D red/cyan anaglyph glasses to see the stereo effect. 


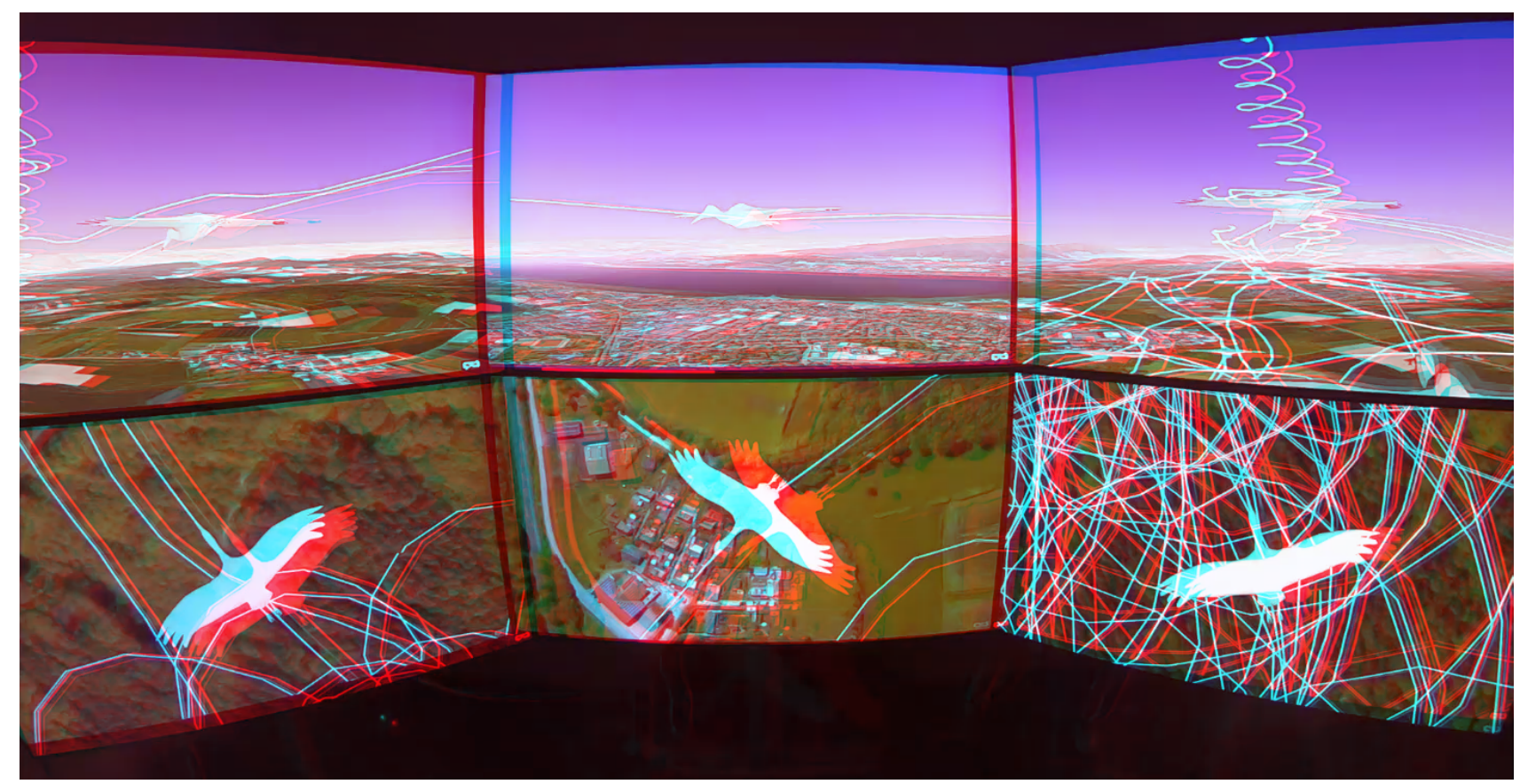

Figure 8. Bird Visualization - Scenario 3 Birds: Three storks, each shown from side and top perspective. The stork in the center is the leading stork, that is the stork at the front. All monitors are used here in 3D mode. Please use S3D red/cyan anaglyph glasses to see the stereo effect.

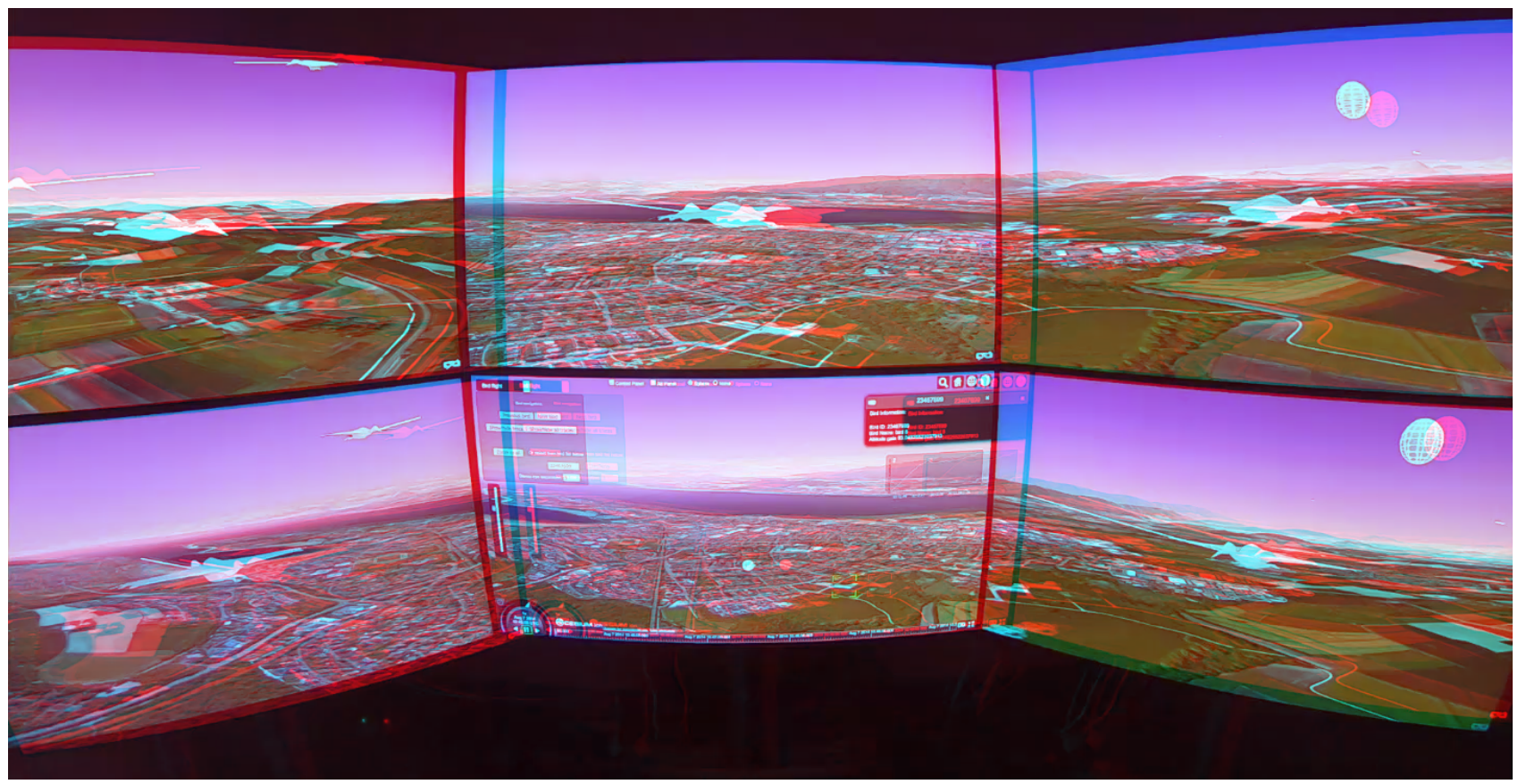

Figure 9. Bird Visualization - Scenario 5 Birds: Five birds, each one shown from side perspective. In the bottom center, the overview showing all animals. All monitors are shown in 3D mode except the center-bottom one which is in 2D mode. As the displays are used with passive 3D glasses, the 3D TVs can be used in $2 D$ as well as $3 D$ mode. Please use S3D red/cyan anaglyph glasses to see the stereo effect. 


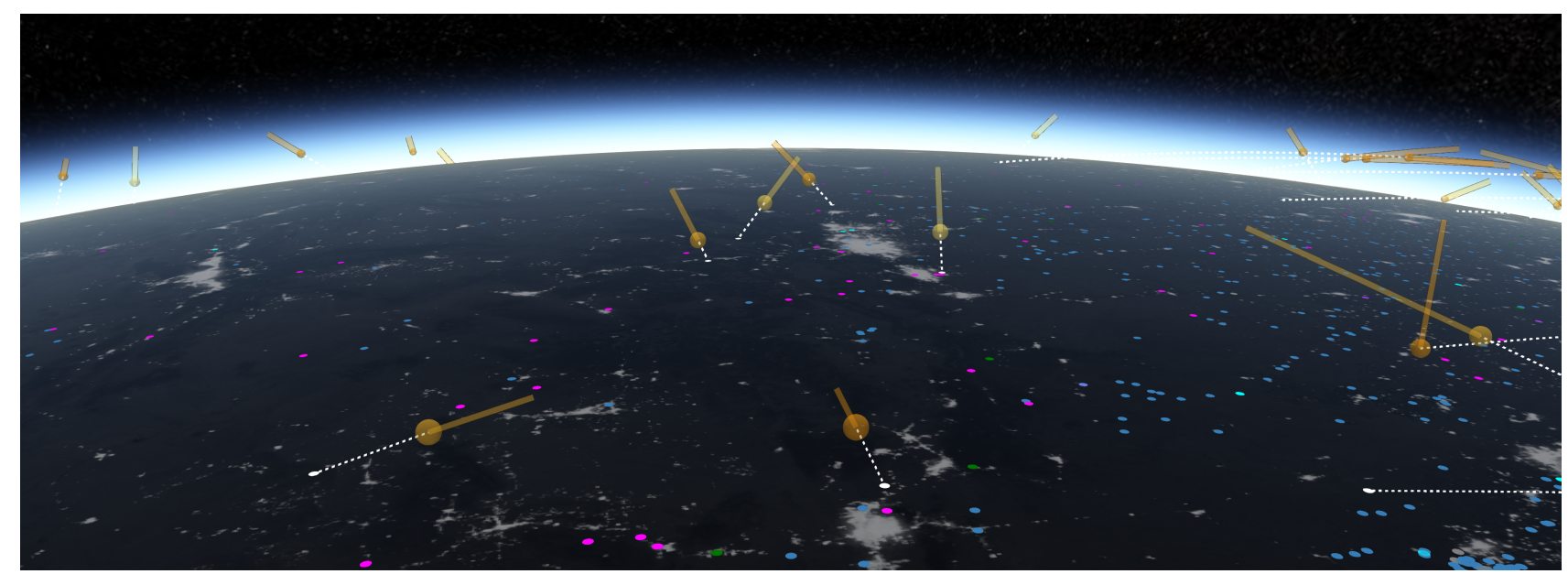

Figure 10. $3 D$ visualization of a subset of fireballs and meteorites from 2012 until 2013. Fireballs are depicted as glyphs with orange shades encoding the stellar magnitude, and length encoding their observed duration and extended trajectory. A white dot encodes the potential fireball impact on the ground. Equally-sized dots are used to depict the meteorites on the ground.

The center display column was directly in front of the viewer, and the two display columns to the left and to the right were placed in an angle of $135^{\circ}$ and $-135^{\circ}$ vice versa to the main column, see also Fig. 12 The six 3D TVs were LG 55EF9509 equipped with 55" displays (display height: $70.1 \mathrm{~cm}$ ). The bottom TVs were placed at a height of $67 \mathrm{~cm}$, the top row in a height of $136.8 \mathrm{~cm}$. In this way, the viewing position was optimized for people at average size of around $1.85 \mathrm{~m}$ for $2 \mathrm{D}$ as well as $3 \mathrm{D}$ viewing.

Two evaluation runs were performed:

1. The first one was static, as shown in Fig. 11, each of the six monitors showed the same perspective.

2. The second one used the same scene but started an animation. As the content of each of the six monitors started at the same time and were synchronized, each of them showed the same perspective at the same time. However, the animation was running, therefore, the perspectives were constantly changing while the bird was flying along its trajectory.

For the evaluation, Cesium was run with the web browser Firefox 65.0.1 (64 bit) on Windows 10. Running Cesium in six browser instances using $4 \mathrm{~K}$ resolution lead to a frame rate of less than 1 FPS which was not usable for our analysis. Therefore, during our evaluation the 3D visualization in Cesium ran smoothly in HD resolution mode while HTML elements on the screen - like GUI buttons and information etc. - were shown in full $4 \mathrm{~K}$. Cesium with the Bird Visualization was switched to fullscreen sideby-side stereo mode. As previously described, the 3D TVs are using circular polarization in combination with FPR technology of LG. Therefore, the left/right perspective of the bottom row had to be inverted. In addition, the 3D TVs had to be switched to 3D mode, transforming the side-by-side image to a polarized image.

The Tiled S3D Display Wall was evaluated by sixteen Bachelor, Master and $\mathrm{PhD}$ students from different departments: computer science, biology, economics, history, philosophy, education, law, as well as physics. The body heights varied between 1.62 and $1.95 \mathrm{~m}$ (average $1.82 \mathrm{~m}$ ). Fig. 13 shows the results of all participants. To be able to differentiate between different body heights and to contrast them with the complete group of 16 participants, three subgroups were created: Group 1 of the smaller participants, smaller than $1.80 \mathrm{~m}$, as can be seen in Fig. 14 (body heights: 1.62, $1.68,1.73$, and $1.78 \mathrm{~m}$ ). The third group is composed of two people who are larger than $1.90 \mathrm{~m}$ Fig. 16 (body heights: 1.93, and $1.95 \mathrm{~m}$ ). In between, the second group consisted of people with average height in our test set, ranging from 1.80 to $1.90 \mathrm{~m}$ in Fig. 15 (body heights: 1.80, 2x1.82, 2x1.83, 1.84, 1.85, 2x1.86, and $1.89 \mathrm{~m})$.

The participants had the task to iteratively visit squares I. to XVIII., stand up straight at the particular position, and rate the S3D effect of every single TV. There were only three options:

- Positive (Value: 1.0, Color: Green)

The stereo effect was well perceived, no eye strain.

- Neutral/Problematic (Value: 0.0, Color: Yellow) The stereo effect can still be perceived, but there might be a little bit eye strain and/or relatively strong ghosting.

- Negative (Value: -1.0, Color: Red)

The stereo effect is extremely poor, both images just seem to overlay each other and there might be strong eye strain when looking at the screen.

We did not want to give participants a more granular scale: In case they were not satisfied with the 3D effect, this should directly lead to dissatisfactory ratings.

Each of the evaluation sheets - with Fig. 13 showing the results of all participants - contain three subsections, each for static $(\mathrm{a}-\mathrm{c})$ and animation $(\mathrm{d}-\mathrm{e})$ mode:

- a/d) Evaluation of Viewing Positions:

Each segment shows the six-monitor composition of the squares I. - XVIII. The two numbers at the right side show the average numbers of each row. The top right number besides the square number indicator shows the overall average value of this square segment. The three signs,+- , and $o$ are explained in the next section. The average rating of all segments is given at the top right corner together with the average height of all involved participants. The color scheme 
is following the previously-mentioned positive/green over yellow/neutral towards negative/red color scale.

- b/e) Overview - Evaluation of Viewing Positions:

The Overview ratings are based on the criteria described below.,,+- and $o$ are color-encoded by green, yellow and red, respectively. This approach has the intention to exclude all sub-optimal viewing positions as the audience expects a good 3D experience and otherwise will be unsatisfied.

- c/f) 3D Effect Rating:

The table gives an overview of the ratings given by the evaluation attendees after using TS3DW. It differentiates between Stereo Quality, Parallel Usage of different monitors, Potential for bird visualization, as well as issues with Refocusing during the evaluation.

For the subsections b/e of the evaluation sheets, the following criteria were applied to generate the overview visualizations of the viewing positions:

- Positive (Value: 1.0, Color: Green)

- No negative entries

- No negative rows

- Neutral/Problematic (Value: 0.0, Color: Yellow)

- Negative entries

- No negative rows

- Negative (Value: -1.0, Color: Red)

- Negative entries

- Negative rows

Again, the intention here was to be quite strict regarding a comfortable S3D experience. Already a minor accumulated negative rating of one monitor can lead to a neutral overall rating, and already a minor accumulated negative rating for a complete row will lead to a negative overall rating in Fig. $13 \mathrm{~b} / \mathrm{e}$ ) and following.

In addition to these ratings, the attendees were asked to generally judge for the static and animation mode of the TS3DW

- the overall quality of the stereoscopic effect,

- if the parallel usage of six 3D TVs side-by-side was usable,

- if they can see its potential for ornithology, and

- if it was problematic to refocus the eyes during its usage.

See Fig. 13 c/f) for examples and for the different five-point rating scales.

\section{Results and Discussion}

16 students participated in the evaluation. First, we analyzed the data of the complete set of attendees (Fig. 13). Then, it was decided to subdivide the group into three sub groups to be able to differentiate between different body height groups:

- 4 Participants with minimal height $<1.80 \mathrm{~m}$ (Fig. 14,

- 10 Participants with height $\geqslant 1.80 \mathrm{~m}$ and $<190 \mathrm{~m}$ (Fig. 15,

- 2 Participants with height $\geqslant 1.90 m$ (Fig. 16.

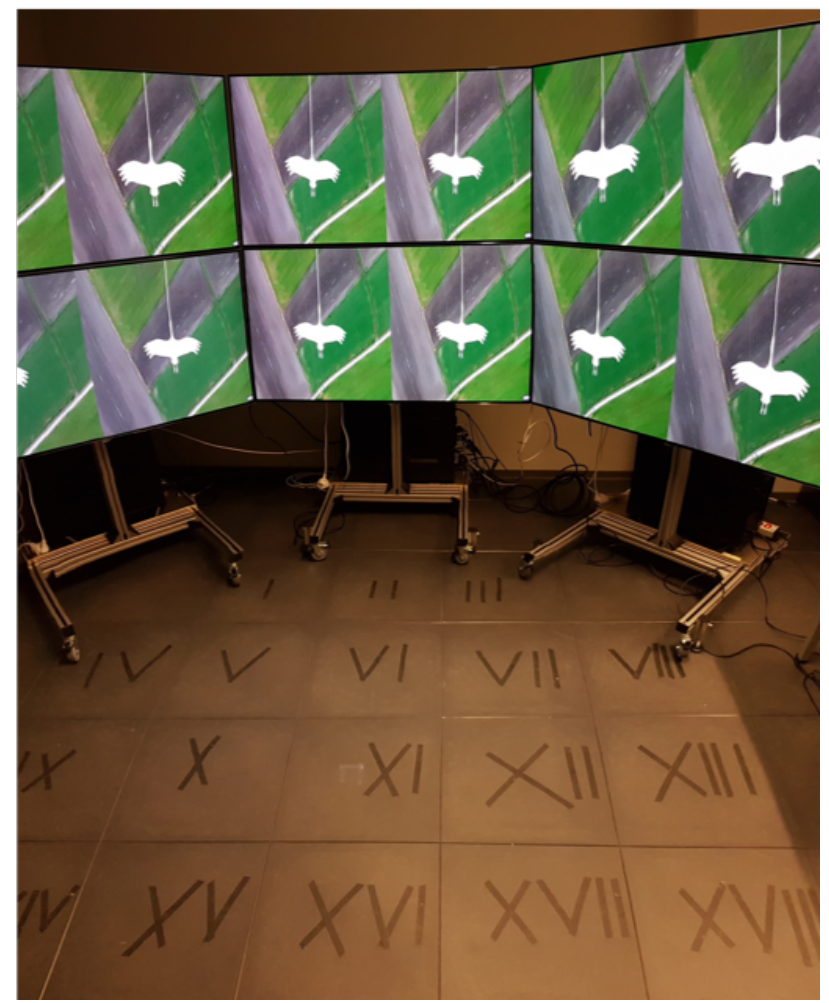

Figure 11. The TS3DW setup used for the evaluation. The sketch is shown in Fig. 12

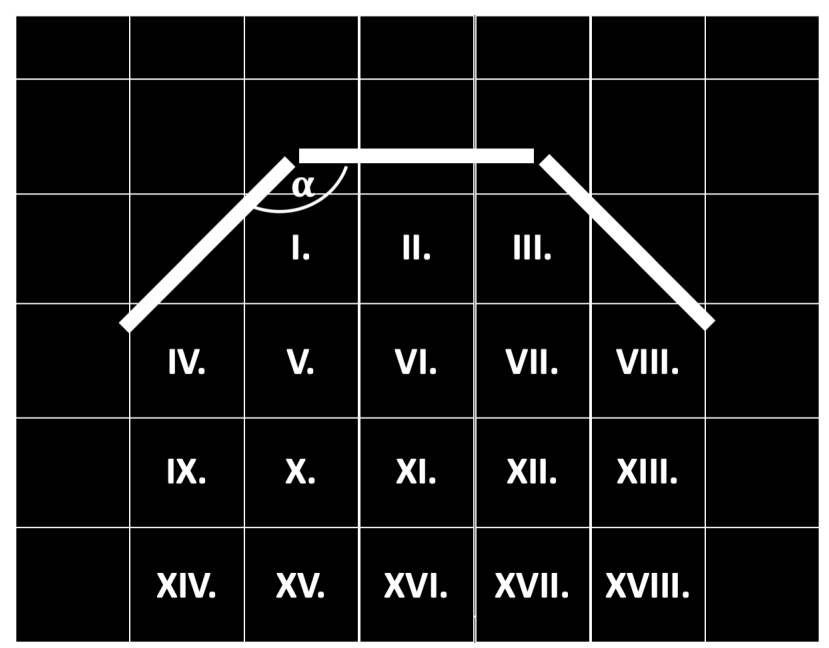

Figure 12. TS3DW evaluation sketch. Each square has a size of $0.5 \times 0.5 \mathrm{~m}^{2}$. The angle $\alpha$ between the displays is $135^{\circ}$. See Fig. 11 for photo. 

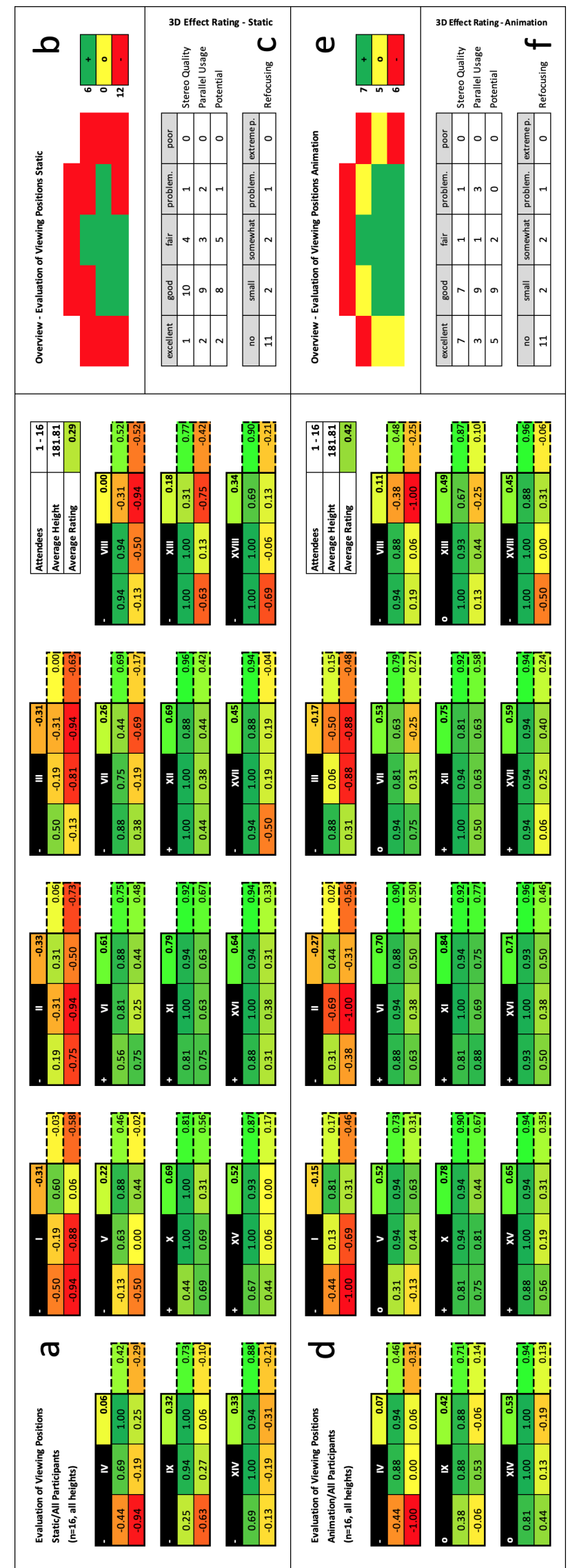

Figure 13. Evaluation of TS3DW Viewing Positions of all Participants: 16 Participants.
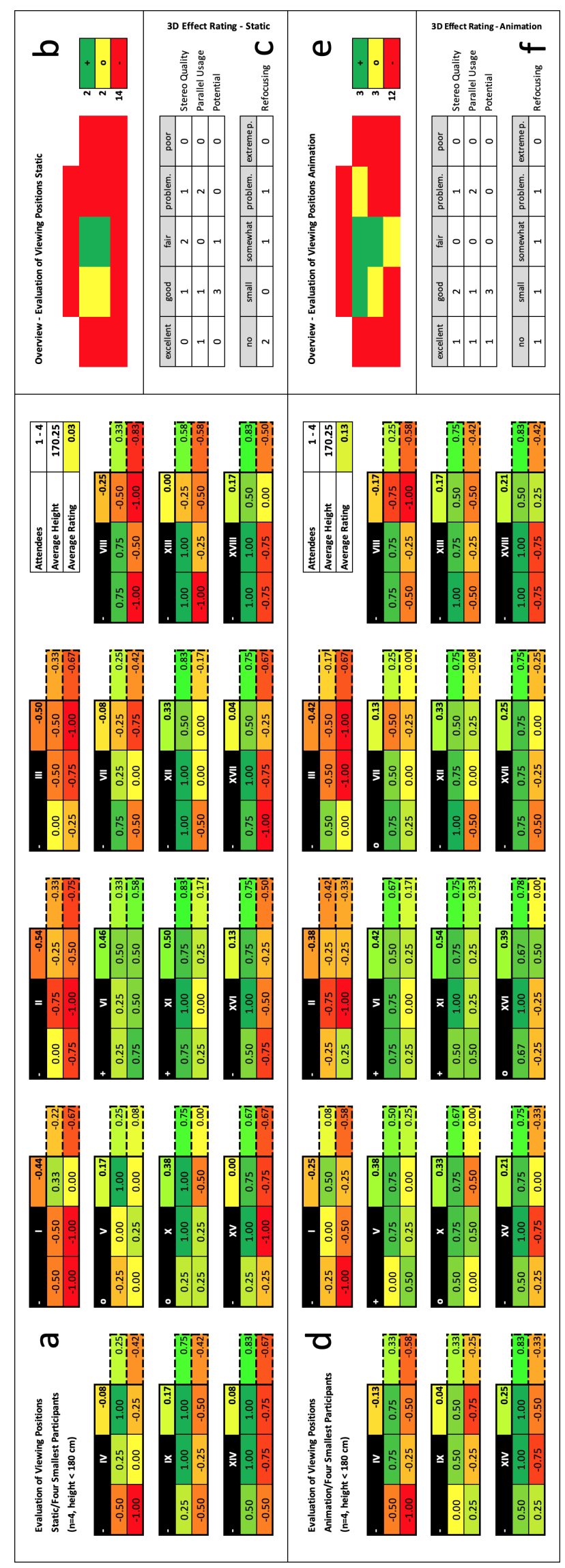

Figure 14. Evaluation of TS3DW Viewing Positions of 4 Participants with minimal height $<130 \mathrm{~m} \mid \mathrm{n}$ ternational Symposium on Electronic Imaging 2019 Stereoscopic Displays and Applications XXX 


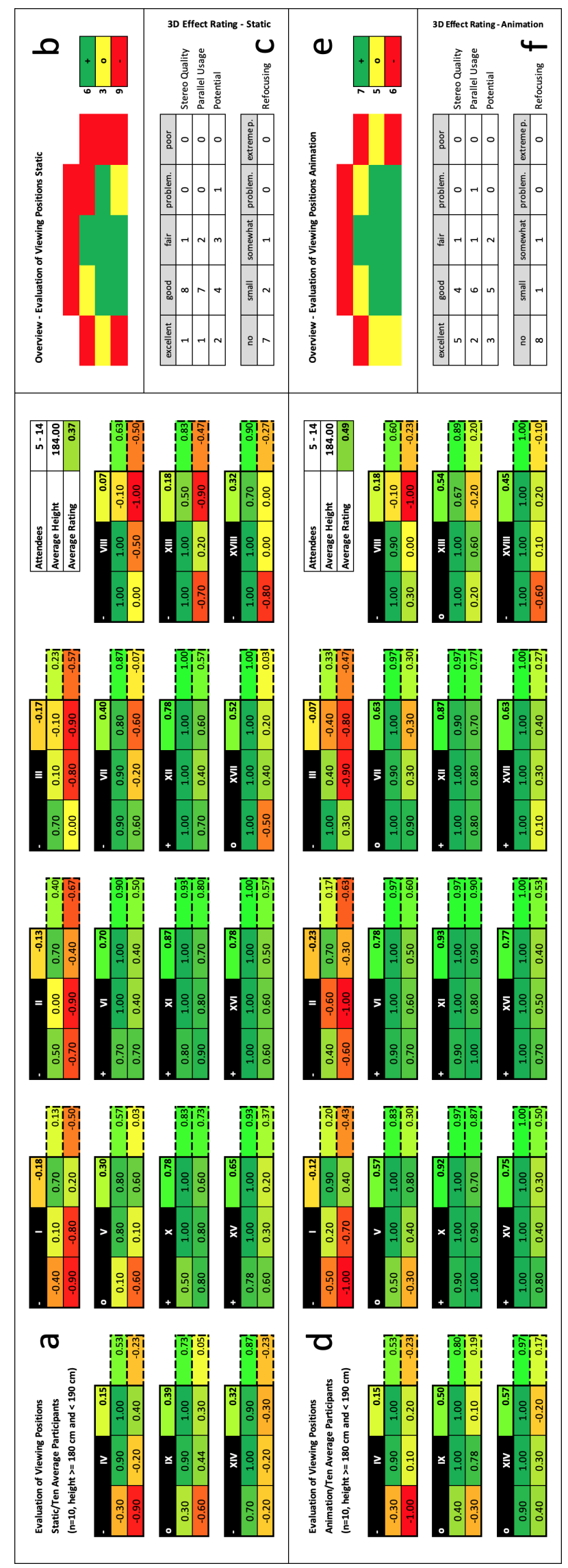

Figure 15. Evaluation of TS3DW Viewing Positions of 10 Participants with

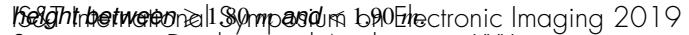
Stereoscopic Displays and Applications XXX
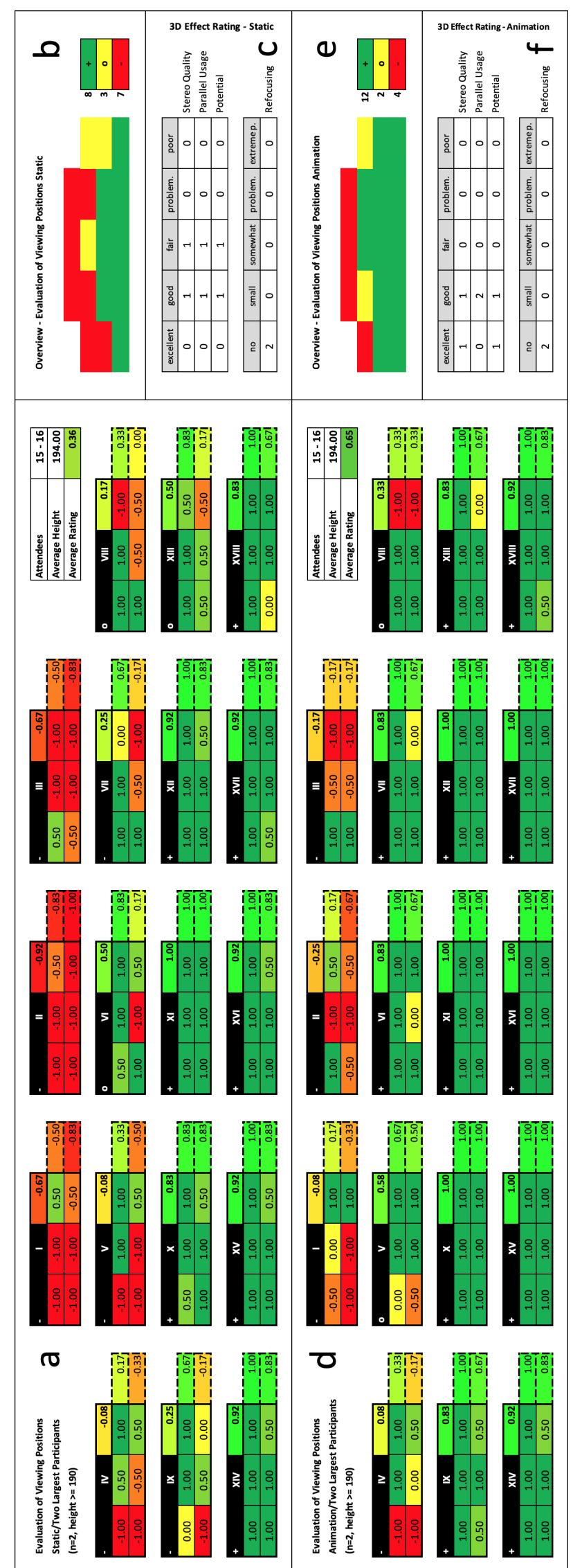

Figure 16. Evaluation of TS3DW Viewing Positions of 2 Participants with height $\geqslant 1.90 \mathrm{~m}$. 


\section{All Participants}

First, we investigated the accumulated data of the sixteen participants, with an average size of $1.82 \mathrm{~m}$ - varying from 1.62 to $1.95 \mathrm{~m}-$ as visible in Fig. 13

In Fig. 13 the section a/b/Evaluation of Viewing Positions shows the Average rating of the static and the animation mode: 0.29 and 0.42 (on a scale from $-1 /$ worst to $+1 /$ best). Obviously, the overall rating is in a positive range, but there have to be some problematic regions included.

Moreover, already here an interesting trend can be seen which was confirmed by examining all subgroups: the animation mode achieved better average ratings than the static mode. It seems to be more comfortable for the eyes to alter the eye's focus during observing a stereoscopic image. In Fig. $13 \mathrm{c} / \mathrm{f}$ the $3 D$ Effect Ratings are shown: 11 participants did not have any problems with refocusing between different monitors, two had minor problems, two had somewhat problems and only one had serious problems. The person who gave the worst rating here also noted that the birds were never clearly visible and that six monitors were too much input info. Obviously, this person had problems with using TS3DW or, at least, with the nature of the task of iteratively evaluating all 3D TVs. But an important fact is that this person was with $1.62 \mathrm{~m}$ the smallest participant in body height. Therefore, it was decided to evaluate the group of smaller people separately and contrast their ratings to the larger persons' group.

The Overview of the Evaluation of Viewing Positions in Fig. $13 \mathrm{~b} / \mathrm{e}$ can be used to quickly identify the optimal viewing positions for visitors. The squares directly neighboring the TS3DW monitors are not usable, as indicated by the red color. Also the squares on the left and right border show bad or neutral ratings. This trend can be observed for all following heightdependent evaluations - the trend only alters for the largest participants' group.

However, for the static mode, six positive squares are indicated, and for the animation mode, seven - indicating the number of optimal viewing positions. From these numbers can be deduced that six to seven people in parallel could use TS3DW. The position in the front could be used, e.g., by an instructor who is also doing the navigation and discussing the scenarios with the audience.

Another trend visible here is the fact that the bottom row of monitors mostly achieves worse ratings than the upper row. The reason is that the upper row usually is directly in straight viewing direction of the user, whereas the bottom row is below the straight sight of the user.

The best viewing position/sweet spot is identified as square XI. with the values of 0.79 and 0.84 on average for all 16 participants. Also, for all subgroups, this position will maintain its pole position. Only for the largest participants there will be more squares featuring the same positive rating.

\section{Small Participants}

As previously mentioned, the results illustrated in Fig. 13 showed some outliers. Therefore, body height-dependent analysis of the results was performed. First, the smallest participant in the range from $1.62 \mathrm{~m}$ to $1.78 \mathrm{~m}$ were analyzed (average height $1.70 \mathrm{~m}$ ).

Fig. $14 a / b$ shows the worst average rating of the static and animation mode: 0.03 and 0.13 . Obviously, the overall rating is quite bad, close to mediocrity - which means that the use of this system is accompanied by an uncomfortable feeling for the users.

The Overview Ratings in Fig. 14 b/e show only two and three optimal viewing positions, respectively. The system seems to be only usable by a single person of this body height. Especially the bottom rows indicate here problematic viewing experiences.

Also, the ratings regarding Stereo Quality, Parallel Usage and Refocusing in Fig. $14 c / f$ are quite fair.

\section{Average Participants}

The ten average size participants, ranging in size from $1.80 \mathrm{~m}$ to $1.89 \mathrm{~m}$ (average height: 1.84) in Fig. 15 show very similar ratings to the overall ratings of all 16 participants, having a slightly increased overall rating. Especially for the static mode there are three additional neutral viewing positions.

Another important difference is that the 3D Effect Ratings in Fig. $15 c / f$ do only show a single problematic entry - all other entries are concentrated around the rating "good". The other four (static) and three (animation) problematic entries from all 16 participants came from the group of smaller participants in Fig. 14

Also, the average rating improved in comparison to all participant's rating, as shown in Fig. $15 a / d$ : for static the rating is 0.37 , and for the animation 0.49 , respectively.

Another important event that occurred in the average size group was that one person felt a little bit vertigo and had to make a break of five minutes during the evaluation run with the animation. This person was known to be sensible to heights. Although not a comfortable experience for this person, it shows that the S3D effect worked quite good by deceiving the participant's visual perception. However, no participant had to quit the evaluation because of dizziness.

\section{Large Participants}

Finally, the largest attendees were analyzed, with a body height of $1.93 \mathrm{~m}$ and $1.95 \mathrm{~m}$ (average height: $1.94 \mathrm{~m}$ ). Fig. $16 \mathrm{~b} / e$ shows a surprising number of optimal viewing positions: 8 for static and even 12 for animation, and an Average Rating of 0.36 and 0.65. The average rating for the static mode was strongly influenced by a very bad rating for squares directly in front of the monitors.

Based on the rating of these two attendees, up to 12 visitors could use the system in case they are larger than $1.90 \mathrm{~m}$. But it has to be said that two attendees are the smallest subgroup elucidated here and that the results have to be judged with care. However, the positive tendency is visible, especially in comparison to the small participants. To double check this result, we extended the data set by another two next-larger participants. The result did not drastically alter from the smaller two-group: only one positive entry for the static mode changed to neutral (data not shown here).

\section{TS3DW Alignment}

Based on the discussed results, it can be stated that the TS3DW system was optimally aligned for the larger participants with a body height of around $1.90 \mathrm{~m}$. Also, for the average participants with a body height of $1.84 \mathrm{~m}$ there were six to seven optimal viewing positions identified. But obviously, TS3DW was not optimally aligned for the use with smaller persons, here with an average height of $1.70 \mathrm{~m}$. Height differences of more than $30 \mathrm{~cm}(1.62 \mathrm{~m}$ to $1.95 \mathrm{~m}$ ) obviously were not taken into account during the initial alignment of the system. 
An obvious solution would be now to move the complete monitor system 10 to $20 \mathrm{~cm}$ down. The viewing experience for the average height group should improve slightly, and the experience for the small height group should drastically improve. On the other hand, the larger persons will get a reduced experience quality.

Still, this might not be the final solution, as body sizes might drastically vary, especially if children are joining the presentation. Therefore, we suggest the following procedure:

In our case, square XI. was identified as the optimal viewing position. Examining Fig. 12 the reason becomes apparent: the distances to all screens show highest similarity in this configuration. This is the starting point for configuring TS3DW.

1. Identify the viewing ranges, by e.g. using a latter or chair to test different heights (while trying to avoid changing the position inside the plane).

2. Identify the maximal and minimum height were the S3D effect is still perceivable (in our rating schema: positive or neutral).

3. Now estimate the maximum and minimum body heights of visitors.

4. Finally, change the vertical alignment of the 3D TVs accordingly, so that all or most visitors can optimally perceive the S3D effect.

Especially in case height differences are larger than the tolerance of the system, other approaches could be used: visitors could be provided with platform shoes, or there could be a special small platform with a height variance of 10 to $20 \mathrm{~cm}$ (of course this could be dangerous for visitors overseeing this platform). Or in case small children visit the system with their parents, children could be offered special S3D glasses with inverted glasses, so that the S3D effect is inverted for their low viewing positions.

\section{Conclusion and Outlook}

Here, we have discussed the Tiled S3D Display Wall (TS3DW). This system consists of a number of 3D TVs using passive circularly polarized TFR technology. Our system consists of three mobile columns with two monitors each. Similar to many other passive 3D TVs, the positioning of the TVs had to be optimized to provide an appropriate $3 \mathrm{D}$ experience.

We showed here that the setup can be comfortably used by a group of up to seven people in case the group is composed of people with an average size around $1.84 \mathrm{~m}$. On the other hand we also showed that the system was not optimized for the use with people of smaller body height below $1.80 \mathrm{~m}$. Therefore, we proposed a procedure to optimize the vertical alignment of the system.

In addition, we discussed two application cases: bird visualization and meteor visualization. Different usage scenarios were introduced. During the evaluation using the bird animation, the potential of S3D visualization for ornithology was rated by 14 from 16 participants as excellent or good. Also, most participants did not have any problems using six 3D TVs in parallel. However, two participants had problems with this setup up to small dizziness feelings.

Based on our evaluation it can be stated that most systems, such as the SunCAVE and the Wave, will have similar problems.

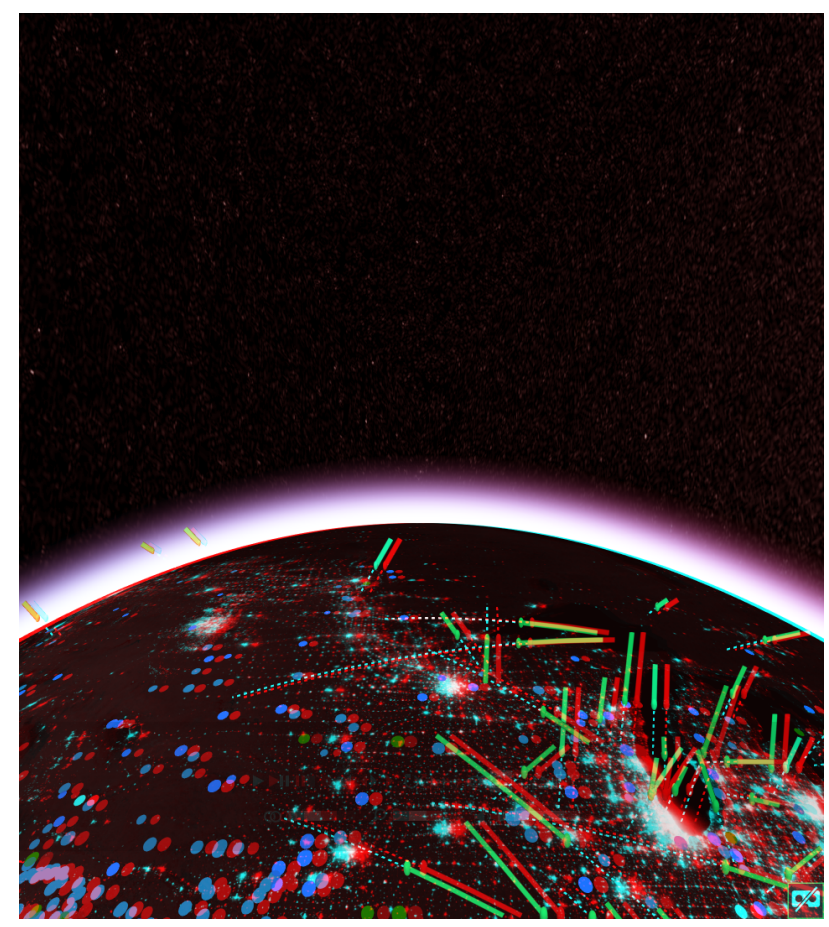

Figure 17. Distant view of fireballs entering into the Atmosphere and meteors on the ground. Please use S3D red/cyan anaglyph glasses to see the stereo effect.

An important advice based on our results is that the monitor system should be mobile in the plane as well as along the vertical axis, so that the height and the angle towards each other can easily be adjusted.

The Bird Visualization as well as the Meteor Visualization systems will be further extended towards Immersive Analytics applications [28]. Based on the results of this paper, we can now start to work with our collaboration partners in this environment using the seven to six optimal viewing positions.

As the commercial 3D TV manufacturers, such as LG, have stopped to produce 3D TVs, we are hoping that soon 3D TVs will be produced again so that similar systems can be constructed in the future. More than 20,000 people have already signed an online petition asking LG to revive the $3 \mathrm{D}$ option on their future OLED TVs [29].

\section{Acknowledgments}

This work has been partially funded by the Deutsche Forschungsgemeinschaft (DFG, German Research Foundation)

- Projektnummer 251654672 - TRR 161 (Project D04),

- as well as within Priority Research Program 1894 (DFG SPP VGI).

Thanks for the construction of the monitor stand go to Mr. Ekkehard Moser and his colleagues of the University of Konstanz' workshop.

The bird migratory data was kindly provided by the Max Planck Institute for Ornithology, Radolfzell: thanks go to Wolfgang Fiedler, Andrea Flack, Máté Nagy, Michael Quetting, Kamran Safi, and Martin Wikelski. For more information see [13]. 
The 3D videos and images were taken with GoPro® 7 Cams provided by Johannes Zagermann of the Konstanz HumanComputer Interaction group of Harald Reiterer.

Additional thanks go to the Blender Foundation for providing the Blender 3D Modeling suite which was here used for creating the initial sketch of TS3DW as seen in Fig. 1 Blender is available from https://www.blender.org

Stereoscopic red-cyan encoding was done with Peter Wimmer's Stereoscopic Player available from http://3dtv . at

\section{References}

[1] C. Cruz-Neira, D. J. Sandin, T. A. DeFanti, R. V. Kenyon, and J. C. Hart, The CAVE: audio visual experience automatic virtual environment, Communications of the ACM, 35(6), pp. 64-72 (1992).

[2] A. Febretti, A. Nishimoto, T. Thigpen, J. Talandis, L. Long, J., D. Pirtle, T. Peterka, A. Verlo, M. Brown, and D. Plepys, CAVE2: a hybrid reality environment for immersive simulation and information analysis., in IS\&T/SPIE Electronic Imaging, pp. 864903-864903-12 (2013).

[3] blender.org - Home of the Blender project - Free and Open 3D Creation Software, https://www.blender.org (last accessed on 2019-02-15).

[4] M. Cordeil, T. Dwyer, K. Klein, B. Laha, K. Marriot, and B. H. Thomas. Immersive Collaborative Analysis of Network Connectivity: CAVE-style or Head-Mounted Display? IEEE Transactions on Visualization and Computer Graphics, 23(1), pp. 441-450 (2016).

[5] B. Sommer, A. Hamacher, O. Kaluza, T. Czauderna, M. Klapperstck, N. Biere, M. Civico, B. Thomas, D. G. Barnes, and F. Schreiber. Stereoscopic Space Map - Semi-immersive Configuration of 3Dstereoscopic Tours in Multi-display Environments. Electronic Imaging, Proceedings of Stereoscopic Displays and Applications XXVII, 2016(5), pp. 1-9 (2016).

[6] T. A. DeFanti, G. Dawe, D. J. Sandin, J. P. Schulze, P. Otto, J. Girado, F. Kuester, L. Smarr, R. Rao., The StarCAVE, a third-generation CAVE and virtual reality OptIPortal. Future Generation Computer Systems, 25(2), pp. 169-178 (2009).

[7] T. A. DeFanti, D. Acevedo, R. A. Ainsworth, M. D. Brown, S. Cutchin, G. Dawe, K.-U. Doerr, A. Johnson, C. Knox, and R. Kooima, The future of the CAVE, Central European Journal of Engineering, 1(1), pp. 16-37 (2011).

[8] R. Kays, M. C. Crofoot, W. Jetz, M. Wikelski, Terrestrial animal tracking as an eye on life and planet, Science, 348(6240), pp. aaa2478 (2015).

[9] N. Greffard, F. Picarougne, and P. Kuntz, Visual community detection: An evaluation of 2D, 3D perspective and 3D stereoscopic displays, International Symposium on Graph Drawing, pp. 215-225 (2011).

[10] J. P. McIntire, P. R. Havig, and E. E. Geiselman. What is 3D good for? A review of human performance on stereoscopic 3D displays, Head- and Helmet-Mounted Displays XVII, 8383, pp. 83830X-1-13 (2012).

[11] B. Sommer, C. Bender, T. Hoppe, C. Gamroth, L. Jelonek, Stereoscopic cell visualization: From mesoscopic to molecular scale, Journal of Electronic Imaging, 23(1), pp. 011007 (2014).

[12] M. Wikelski, R. Kays, Movebank: archive, analysis and sharing of animal movement data. World Wide Web electronic publication: https://movebank.org Accessed on January 302019 (2014).

[13] K. Klein, B. Sommer, H. T. Nim, A. Flack, K. Safi, M. Nagy, S. P. Feyer, Y. Zhang, K. Rehberg, A. Gluschkow, M. Quetting,
W. Fiedler, M. Wikelski, F. Schreiber, Fly with the flock: immersive solutions for animal movement visualization and analytics, Journal of the Royal Society Interface, 16(153), 20180794 (2019).

[14] J. M. Hilbe, J Riggs, B. D. Wandelt, R. S. de Souza, E. EO Ishida, J. Cisewski, V. Surdin et al. Life, the universe, and everything. Significance, 11(5), pp. 48-75 (2014).

[15] American Meteor Society, Ltd., https://www.amsmeteors. org/ (last accessed on 2019-02-08).

[16] M.S. Dias, J. d'Alpuim, P. Caetano, Galactica, a Digital Planetarium for Immersive Virtual Reality Settings, International Journal of Creative Interfaces and Computer Graphics (IJCICG), 7(1), pp. 19-39 (2016).

[17] A. Szalay, J. Gray, The world-wide telescope, Science, 293(5537), pp. 2037-2040 (2001).

[18] F. Chreau, R. Spearman, J. Meuris, J. Gajdosik, Stellarium, https : //sourceforge.net/projects/stellarium/ (last accessed on 2019-02-15).

[19] Asterank, http://www.asterank.com/ (last accessed on 201902-08).

[20] Stuff in Space, http://stuffin.space/ (last accessed on 201902-08).

[21] Meteor showers as seen from space, https://www. meteorshowers.org (last accessed on 2019-02-08).

[22] Bolides, http://bolid.es/ (last accessed on 2019-02-08).

[23] G.-Y. Lee, S.-H. Lee, H.-J. Kwon, K.-I. Sohng, Visual perceptualbased film-type patterned retarder three-dimensional image representation, Optical Engineering, 54(10), pp. 103106 (2015).

[24] cesiumjs API Reference. http://cesiumjs.org/refdoc.html (last accessed on 2019-02-15).

[25] A. J. Woods, How are crosstalk and ghosting defined in the stereoscopic literature? Proc. SPIE 7863: Stereoscopic Displays and Applications XXII, pp. 78630Z.1-12 (2011).

[26] R. Weinzierl, G. Bohrer, B. Kranstauber, W. Fiedler, M. Wikelski, A. Flack, Wind estimation based on thermal soaring of birds, Ecology and Evolution, 6(24), pp. 8706-8718 (2016).

[27] A. Flack, W. Fiedler, M. Wikelski, Data from: Wind estimation based on thermal soaring of birds. Movebank Data Repository. doi:10.5441/001/1.bj96m274 (2017).

[28] B. Sommer, D. Barnes, S. Boyd, T. Chandler, M. Cordeil, K. Klein, T. D. Nguyen, H. Nim, K. Stephens, D. Vohl, S. Wang, E. Wilson, J. McCormack, K. Mariott, F. Schreiber, 3D-Stereoscopic Immersive Analytics Projects at Monash University and University of Konstanz, IS\&T Electronic Imaging: Stereoscopic Displays and Applications XXVIII Proceedings, IS\&T, Springfield, VA (2017).

[29] Change.org: LG - Please revive 3D on a 2018 OLED TV model. https://www.change.org/p/ lg-please-revive-3d-on-a-2018-oled-tv-model (last accessed on 2019-02-15). 